\title{
Design of a Recognizing System for Vehicle's License Plates with English Characters
}

\author{
Xiong Xing ${ }^{1}$, Byung-Jae Choi ${ }^{*}$, Seog $\mathrm{Chae}^{2}$ and Mun-Hee $\mathrm{Lee}^{3}$ \\ ${ }^{1}$ School of Electronic Engineering, Daegu University \\ E-mail: xiongxing@nate.com, bjchoi@daegu.ac.kr \\ 2 School of Electronic Engineering, Kumoh University \\ 3 Daegu Gyeongbuk Development Institute
}

\begin{abstract}
In recent years, video detection systems have been implemented in various infrastructures such as airport, public transportation, power generation system, water dam and so on. Recognizing moving objects in video sequence is an important problem in computer vision, with applications in several fields, such as video surveillance and target tracking. Segmentation and tracking of multiple vehicles in crowded situations is made difficult by inter-object occlusion.

In the system described in this paper, the mean shift algorithm is firstly used to filter and segment a color vehicle image in order to get candidate regions. These candidate regions are then analyzed and classified in order to decide whether a candidate region contains a license plate or not. And then some characters in the license plate is recognized by using the fuzzy ARTMAP neural network, which is a relatively new architecture of the neural network family and has the capability to learn incrementally unlike the conventional BP network. We finally design a license plate recognition system using the mean shift algorithm and fuzzy ARTMAP neural network and show its performance via some computer simulations.
\end{abstract}

Key Words : License plate recognition, Image processing, Mean shift algorithm, Fuzzy ARTMAP, Neural Network

\section{Introduction}

During the past few years, intelligent transportation systems (ITSs) have had a broad impact in people's lives as they have improved transportation safety and mobility and enhanced productivity through the use of advanced technologies. These systems are divided into intelligent infrastructure systems and intelligent vehicle systems. In this paper, a computer vision and character recognition algorithm for a license plate recognition (LPR) is presented. The system presented is suitable as a core component for use in intelligent infrastructures like electronic payment (toll payment, parking fee payment) systems, and freeway and arterial management systems for traffic surveillance. Moreover, as increased security awareness has made the need for vehicle based authentication technologies extremely significant, the proposed system may be employed as an access control system for monitoring of unauthorized vehicles entering private areas.

The license plate remains as the principal vehicle identifier despite the fact that it can be deliberately altered in situations of fraud or replaced (e.g. with a stolen plate). Therefore, ITSs rely heavily on robust LPR systems. The main job is to analyze images obtained from the camera, according to an image processing technique, and then recognize some characters in the plate. Specifically, the contributions are as follows:

Manuscript received Jul. 17, 2009; revised Sep. 10, 2009.

* Corresponding Author
1) a discontinuity preserving smoothing technique named mean shift algorithm used for faster detection of regions of interest (RoI);

2) an algorithmic sequence called Fuzzy ARTMAP is used to recognize some characters.

There are three stages in the recognition process: license plate detection, character extraction and character recognition. They will be presented in detail in the next part.

\section{Related Works}

It is known that a license plate is a pattern composed of several characters that have high distinctive intensities from their background. The high contrast area can be used as a key feature to detect the desired license plate region. In the following, we will describe a mean shift based method to extract the high contrast area and detect the desired license plate area. We then describe how to utilize Fuzzy ARTMAP to recognize some characters in the extracted area. A flowchart of the proposed license plate detection algorithm is shown in Fig.1.

The first stage is to determine a candidate area from real vehicle images where license plate could exist. This stage deals with image acquisition and the pre-processing operation which removes the noise. The filtering operation of this stage brings the image to an appropriate format for the identification stage. To detect the character region of the license plate, the mean shift algorithm and intensity de- 
rivatives are used. The mean shift algorithm is a non-parametric clustering technique which does not require prior knowledge of the number of clusters, and does not constrain the shape of the clusters.

\begin{tabular}{l}
\hline License Plate Detection \\
\hline Mean shift algorithm \\
\hline Character Segmentation \\
\hline Binarization projection \\
\hline Character Recognition \\
\hline Fuzzy ARTMAP \\
\hline
\end{tabular}

Fig. 1 A three stage approach for a license plate recognition

The second stage is a segmentation of extracted license plate. This includes character extraction. In LPR system, a lot of prior information can be utilized such as the size of the license plate, the size of the characters, and the position of the characters on the license plate. In this paper, we use the information such as contour, shape and disposition to locate the characters. We then use the binarization method to process the extracted characters to provide a stable character feature.

The last stage is a character recognition which is one of the core part of our research. This identification stage deals with the optical recognition of the English and numeric characters in the digital image, and the identification of the vehicle number. Actually, it is an OCR system. An excellent survey on OCR is given by Carpenter elent. [7]. In our application, we need to recognize the printed characters which is easier than handwurvten character recognition. In this paper, we introduce the grid feature and artificial neural network to perform the OCR test.

\section{Design of License Plate Recognition System}

The LPR sequence, which is proposed in this paper, consists of two distinct parts. The first one deals with the detection of the RoI, i.e., the license plate. The second part includes operations for the successful segmentation of the license plate characters along with an artificial neural network, which performs the OCR task.

\subsection{Part 1: License Plate Segmentation}

This part consists of preprocessing in order to detect the RoI in ambient illumination conditions. The preprocessing component consists of four tasks: implementation of the mean shift filtering method, delineation of the cluster, elimination of spatial regions smaller than $\mathrm{M}$ pixels, and extraction of the rectangularity and aspect ratio. The discontinuity preserving smoothing of the mean shift algorithm is indicated in Table I.

An image is typically represented as a two-dimensional lattice of $p$-dimensional vectors. The space of the lattice is known as the spatial domain, while gray level, color, or spectral information is represented in the range domain. For both domains, Euclidean metric is assumed. When the location and range vectors are concatenated in the joint spatial-range domain of dimension $d=p+2$, their different nature has to be compensated by proper normalization. Thus the multi-variate kernel is defined as the product of two radially symmetric kernels and the Euclidean metric allows a single bandwidth parameter for each domain

$$
K_{h_{s}, h_{r}}(x)=\frac{C}{h_{s}^{2} h_{r}^{p}} k\left(\left\|\frac{x^{s}}{h_{s}}\right\|^{2}\right) k\left(\left\|\frac{x^{r}}{h_{r}}\right\|^{2}\right)
$$

where $x^{s}$ is the spatial variable, $x^{r}$ is the range variable of a feature vector, $k(x)$ is the common profile used in both of the two domains, and $C$ is the corresponding normalization constant.

In the joint spatial-range domain we used mean shift filtering, each data point is associated to a point of convergence which represents the local mode of the density in the d-dimensional space. Smoothing through replacing the pixel in the center of a window by the average of the pixels in the window blurs the image indiscriminately and removes not only the noise but also salient information. Discontinuity preserving smoothing techniques, on the other hand, reduces the amount of smoothing near abrupt change in the structure adaptively. The procedure continues until all pixels in the image are scanned.

The license plate segmentation operation is implemented on the mean shift filtered images. The original input image is firstly normalized with a normal kernel function, where the bandwidths in the spatial domain and the range domain are denoted as $h_{s}$ and $h_{r}$, respectively. Let $x_{i}$ and $z_{i}$, $j=1,2, \cdots, n$, be the d-dimensional input and filtered image pixels in the joint spatial-range domain, respectively.

Since this process takes into account both the spatial and range information of the image simultaneously, it can achieve a high quality and discontinuity preserving spatial filtering.

After the candidate regions are obtained by applying mean shift filtering, features of each region are to be extracted in order to differentiate the license plate regions from others correctly. Various features have been utilized for this pur tss puuch features include the size of the region, the width and the height of the region, the orientation of the characters, edge inttures, and the tsition of the region puome features, hourhei, can itiondeal with images captured under very specific environmental conditions.

The license plate has a rectangular shape with a predetermined length to width ratio on each kind of vehicle. Due to the viewing angle and uneven or curvy road surfaces, however, license plate images taken from a vehicle image are usually no longer a standard rectangular shape. Under limited distortion, however, license plates in vehicle images can still be viewed as approximately as rectangular shape with a certain aspect ratio. This is the most important shape feature of license plates. 
Table I. Discontinuity Preserving Smoothing

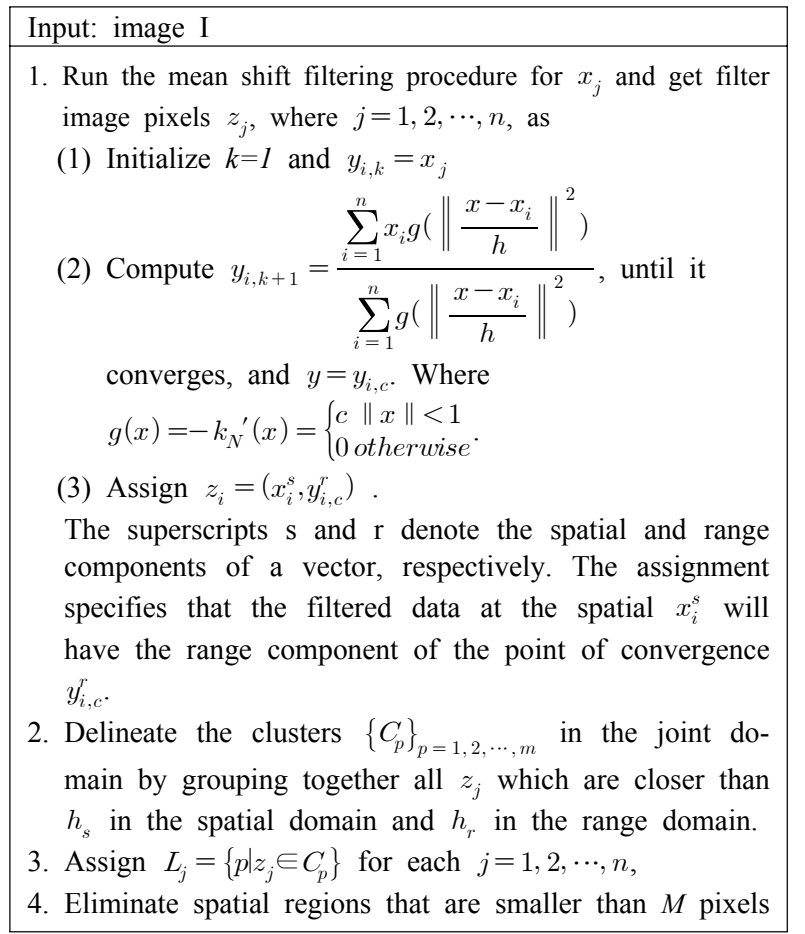

A quick and straightforward method is used to detect the edge of the object region to get its minimum enclosing rectangle (MER). With this method, an edge of RoI is a transition from background to object or vice versa. But for an image that contains a license plate may also include the dynamo and fore-baffle etc, which have very strong horizontal edges. We can see that a vertical edge detector is better than a horizontal edge detector in suppressing horizontal noise.

$$
\begin{aligned}
g_{H}= & \mid[f(i-1, j-1)+2 f(i-1, j)+f(i-1, j+1)] \\
& -[f(i+1, j-1)+2 f(i+1, j)+f(i+1, j+1)] \mid \\
g_{V}= & \mid[f(i-1, j-1)+2 f(i, j-1)+f(i+1, j-1)] \\
& -[f(i-1, j+1)+2 f(i, j+1)+f(i+1, j+1)] \mid
\end{aligned}
$$

Where $f(i, j)$ represents the gray image of the input image after smoothing and normalization, $g_{H}(i, j)$ and $g_{V}(i, j)$ represents the horizontal edge map and vertical edge map, respectively.

The aspect ratio is defined as the ratio of the width to the height of the region's MER. Since the MER of the object region can be computed via rotating the region in the previous section, the dimension of the object's MER can be taken as the width and the height of the region. This ratio is defined by

$$
\text { ratio }=\frac{c_{\max }-c_{\min }+1}{r_{\max }-r_{\min }+1}
$$

where $c$ and $r$ indicates column and row, respectively. The orientation of the object is defined by

$$
\tan \left(2 \theta_{i}\right)=2 \frac{\sum_{r=0}^{N-1} \sum_{c=0}^{N-1} r c I_{i}(r, c)}{\sum_{r=0}^{N-1} \sum_{c=0}^{N-1} r^{2} I_{i}(r, c)-\sum_{r=0}^{N-1} \sum_{c=0}^{N-1} c^{2} I_{i}(r, c)},
$$

Those candidate regions whose measurements fulfill the criteria orientation $<35^{\circ}, 2<$ aspect ratio $<6$, are considered as candidate plate regions.

After applying the above three features to filter the segmented regions, many non-license plate regions can be removed. However, many candidate regions are still left, which have similar rectangularity and aspect ratio features as the license plate regions do, such as the head lights. The edge density is measured in a region $\mathrm{R}$ by averaging the intensities of all edge pixels within the region as

$$
D_{R}=\frac{1}{N_{R}} \sum_{m, n \in R} E(m, n)
$$

where $E(i, j)$ represents the edge magnitude at location(i, $\mathrm{j}$ ), and $N_{R}$ is the number of pixels in region $\mathrm{R}$.

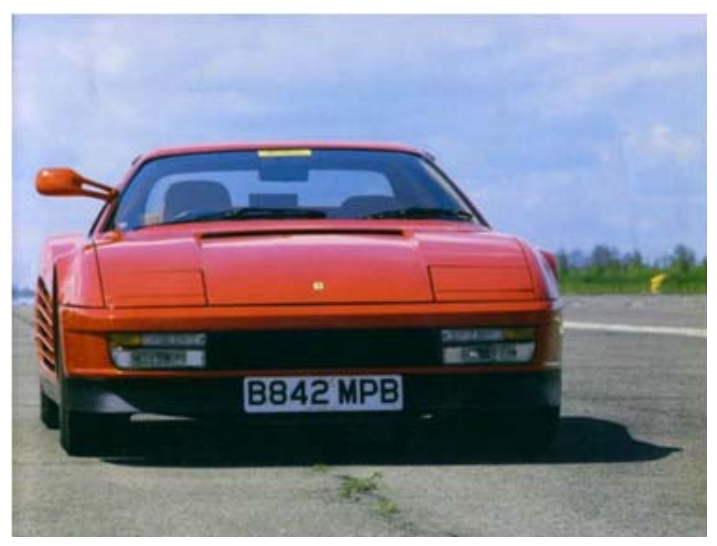

(a)
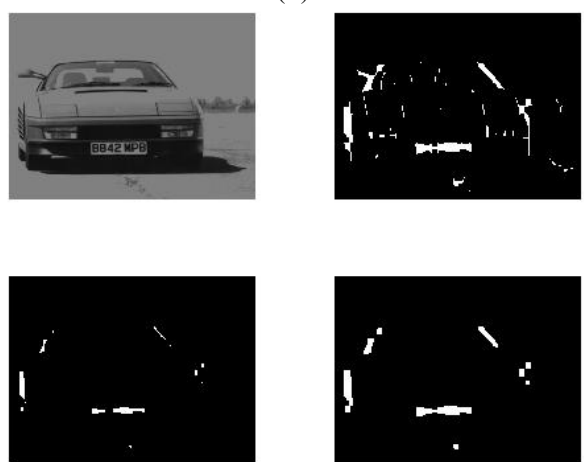

(b)

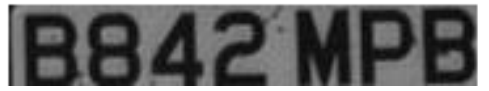

(c)

Fig. 2 Steps for license plate segmentation: (a) Initial image. (b) Results of mean shift algorithm and edge detection. (c) Detected plate region.

\subsection{Part 2: License Plate Processing}

This part consists of the preprocessing and forward step. 
Preprocessing isolates the characters in the license plate image. And the forward step sends those characters to the Fuzzy ARTMAP neural network for identification.

Following the inversion of the resulting image and object labeling, the orientations and heights of connected components (objects) are then calculated. The components whose measurements do not fulfill specific rules (orientation $>75^{\circ}$ and height $>32$ pixels) are deleted. The remaining objects are then forwarded to Fuzzy ARTMAP after a well defined preparation process. And it includes character segmentation calculating the standard deviation of columns and rows, and the transformation of each character to the size of the input vector of the Fuzzy ARTMAP. Fig. 3 presents an example of license plate processing with the proposed algorithm.

\section{B8842 MPB}

Fig. 3 Successful segmentation of the character part

\subsection{Part 3: Character Recognition}

After the Character Segmentation is finished, the next step is to recognize some characters. A combination of neural and fuzzy techniques (Fuzzy ARTMAP Neural Network) is used to guarantee a very low error rate at an acceptable recognition rate. It is widely applied in practice, which will be introduced in the following paragraphs.

ARTMAP (Adaptive Resonance Theory - Mapping) is a class of neural networks that can perform incremental supervised learning. It also performs multi-dimensional mapping in response to binary input vectors presented arbitrarily. The Fuzzy ARTMAP extends the ARTMAP by integrating fuzzy logic into the system, which allows it to accept input vector values between 0 and 1 .

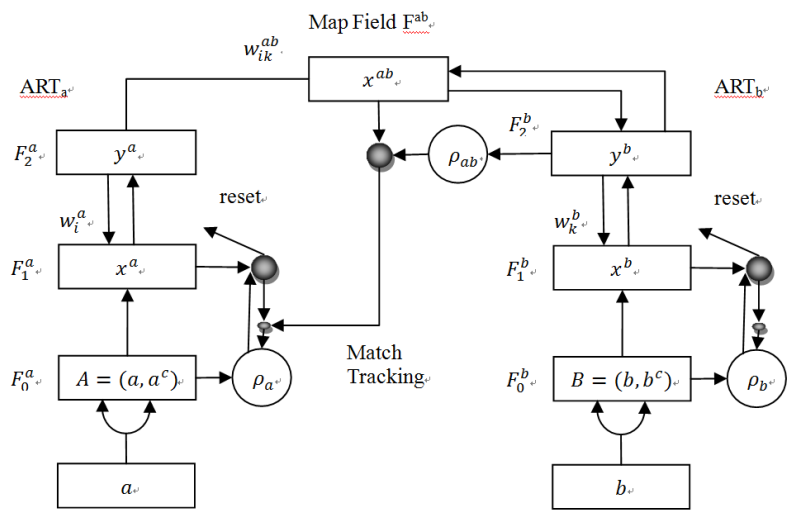

Fig. 4 Fuzzy ARTMAP Architecture

Fuzzy ARTMAP (Fig.4) consists of two fuzzy ART modules $\left(\mathrm{ART}_{\mathrm{a}}\right.$ and $\left.\mathrm{ART}_{\mathrm{b}}\right)$. The parameters of these networks are designated by the subscripts $a$ and $b$, respectively. The fuzzy ART algorithm is summarized in Table II.
Table II. Summary of Fuzzy ART Algorithm

1. ART Field Activity Vector: The ART complement coding pre-processor transforms the $M$ vector a into the $2 M$ vector $A=\left(a, a^{c}\right)$ at the ART field $F_{0}$. A is the input vector to the ARTa field $F_{1}$.

2. Initial Weight Vectors: $w_{j}=1 . w_{j}=\left(w_{j 1}, w_{j 2}, \cdots, w_{j, 2 M_{a}}\right)$ denotes the $F_{2}$ output vector.

3. Category Choice: For each input $\mathrm{I}$ and $F_{2}$ node $j$, the choice function, $T_{j}=\frac{\left|I \wedge w_{j}\right|}{\alpha+\left|w_{j}\right|}$, and the category choice is indexed by $J$, where $T_{J}=\max \left\{T_{j}: j=1 \cdots N\right\}$. When the $J$ th category is chosen, $y_{J}=1$; and $y_{j}=0$ for $j \neq J$.

4. Activity Vector $x$ : $x= \begin{cases}I & F_{2} \text { isn't active } \\ I \wedge w_{J} & \text { Jth } h \text { node of } \mathrm{F}_{2} \text { node is chosen }\end{cases}$

5. Resonance or Reset: If the match function, $\frac{\left|I \wedge w_{J}\right|}{|\hbar|} \geq \rho$, resonance occurs. Then the learning begins. Else $T_{J}$ is set to 0 and a new index $J$ is chosen by category function. The search process continues until $J$ satisfies.

6. Learning: After ending of the searches, the weight vector $w_{J}$ is updated by $w_{J}^{\neq w}=\beta\left(I \wedge w_{J}^{\text {old }}\right)+(1-\beta) w_{J}^{\text {old }}$.

The two Fuzzy ARTs are interconnected by a series of connections between $\mathrm{F}_{2}$ layers of $\mathrm{ART}_{\mathrm{a}}$ and $\mathrm{ART}_{\mathrm{b}}$. These connections form what is called the map field $F_{\mathrm{ab}}$. The map field is used to form predictive associations between categories and to realize the match tracking rule, whereby the vigilance parameter of $\mathrm{ART}_{\mathrm{a}}$ increases in response to a predictive mismatch at $\mathrm{ART}_{\mathrm{b}}$.

The input to $\mathrm{ART}_{\mathrm{a}}$ and $\mathrm{ART}_{\mathrm{b}}$ are in complement code form, which is necessary for the successful operation of fuzzy ARTMAP. For $\mathrm{ART}_{\mathrm{a}}$, if input is $I_{o}=\left(a_{1}, \cdots, a_{M_{a}}\right)$, then

$$
I=\left(a, a^{c}\right)=\left(a_{1}, \cdots, a_{M}, a_{1}^{c}, \cdots, a_{M}^{c}\right)
$$

where

$$
a_{i}^{c}=1-a_{i} \quad 1 \leq i \leq M_{a} \bullet
$$

The $F_{1}^{a}$ activity vector is denoted by $x^{a}=\left(x_{1}^{a}, \cdots, x_{2 M_{a}}^{a}\right)$ and the $F_{2}^{a}$ activity vector with $N_{a}$ number of categories is denoted by $y^{a}=\left(y_{1}^{a}, \cdots, y_{2 N_{a}}^{a}\right) . M_{a}$ and $N_{a}$ are arbitrary. For $\mathrm{ART}_{\mathrm{b}}, O_{o}=\left(b_{1}, \cdots, b_{M_{b}}\right), O=\left(b, b^{c}\right)$. The $F_{1}^{b}$ activity vector is denoted by $x^{b}=\left(x_{1}^{b}, \cdots, x_{2 M_{b}}^{b}\right)$ and the $F_{2}^{b}$ activity vector with $N_{b}$ number of categories is denoted by $y^{b}=\left(y_{1}^{b}, \cdots, y_{N_{b}}^{b}\right) . M_{b}$ and $N_{b}$ are also arbitrary. For the inter-ART modules, the map field $F_{a b}$ output vector is denoted by $x^{a b}=\left(x_{1}^{a b}, \cdots, x_{N_{b}}^{a b}\right)$, and let $w^{a b}=\left(w_{j 1}^{a b}, \cdots, w_{j N_{b}}^{a b}\right)$ denote the weight vector from the j-th $F_{2}^{b}$ node to $F_{a b}$. Vectors $x^{a}, y^{a}, x^{b}, y^{b}, x^{a b}$ are initialized to zero between input presentations.

The inter-ART module $F_{a b}$ is activated whenever any of the $\mathrm{ART}_{\mathrm{a}}$ or $\mathrm{ART}_{\mathrm{b}}$ categories is active. The $F^{a b}$ output 


$$
\begin{gathered}
\text { vector } x^{a b} \text { obeys } \\
x^{a b}=\left\{\begin{array}{cc}
y^{b} \wedge w_{J}^{a b} \quad \text { the ARTa and ARTbis active } \\
w_{J}^{a b} \quad \text { ARTaisactive, ARTbisn'tactive } \\
y^{b} \quad \text { ARTaisn'tactive, and ARTbisactive } \\
0 & \text { the ARTa and ARTbisn'tactive }
\end{array}\right.
\end{gathered}
$$

During the learning phase, the input vector $I_{0}$ is presented to $\mathrm{ART}_{a}$ and the desired output vector $\mathrm{O}_{\mathrm{o}}$ is presented to $\mathrm{ART}_{\mathrm{b}}$. The $\mathrm{ART}_{\mathrm{a}}$ vigilance parameter $\rho_{a}$, equals the baseline vigilance, $\overline{\rho_{a}}$ at the beginning of every input presentation. When Fuzzy ARTMAP receives an input/output $\operatorname{pair}\left(I_{o} / O_{O}\right), \mathrm{ART}_{\mathrm{a}}$ chooses the $\mathrm{J}_{-}$th node of $F_{2}^{a}$ and $\mathrm{ART}_{\mathrm{b}}$ chooses the $\mathrm{K}_{-}$th node of $F_{2}^{b}$. When both $\mathrm{ART}_{\mathrm{a}}$ and $\mathrm{ART}_{\mathrm{b}}$ are active and $x^{a b} \neq 0$, then the input/output pairs are associated with the equation:

$$
w_{j k}^{a b}=\left\{\begin{array}{cc}
1 & j=J \text { and } k=K \\
0 & \text { otherwise }
\end{array}\right.
$$

The $\mathrm{ART}_{\mathrm{a}}$ and $\mathrm{ART}_{\mathrm{b}}$ modules classify the input and desired output vector into categories, then the map field (inter-ART module) makes associations from the $\mathrm{ART}_{\mathrm{a}}$ category to the $\mathrm{ART}_{\mathrm{b}}$ category.

If $x^{a b}=0$, then there is a mismatch. The Inter-ART module triggers a match tracking mechanism, which increases $\rho_{a}$, by a minimum value. And it forces the $\mathrm{ART}_{\mathrm{a}}$ module to search for another category suitable to be associated with the desired output vector. $\rho_{a}$ is then set back to the baseline vigilance parameter, $\bar{\rho}$, for every step of learning trial.

\section{Simulation Results}

To evaluate the efficiency, our method has been tested using MATLAB 2008 over colour images, acquired by a digital camera for real scenes. The test image has been acquired from the front as well as from the rear of vehicles under various illumination and weather conditions (such as sunny, cloudy, daytime, and night time).

There are several types of license plates for the identification of the vehicle. They are not the same in their shape, size, character type, and etc. They even include some background images of tree, building, or etc. These license plates are used to test the performance of the proposed algorithm.

A lot of templates was needed in the proposed system which requires substantial time to make the classifier, but it is essential. Some of the obtained images from the digital camera and binarization results of the license plate are shown in Fig. 5.

In the part of character recognition, the inputs of Fuzzy ARTMAP algorithm are the images of numbers and letters on vehicle license plates. The testing set has 300 characters, around $85 \%$ of the characters have better quality, and the others are all degenerate to different degree.

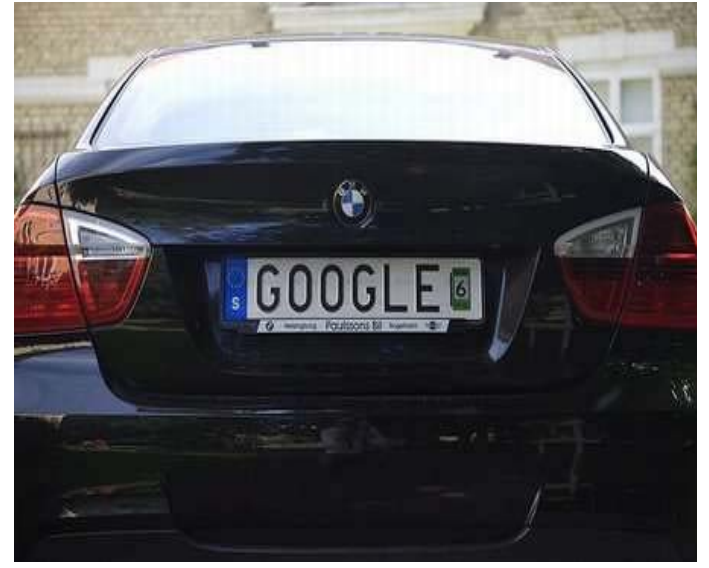

(a)

\section{GOOGLEE}

(b)

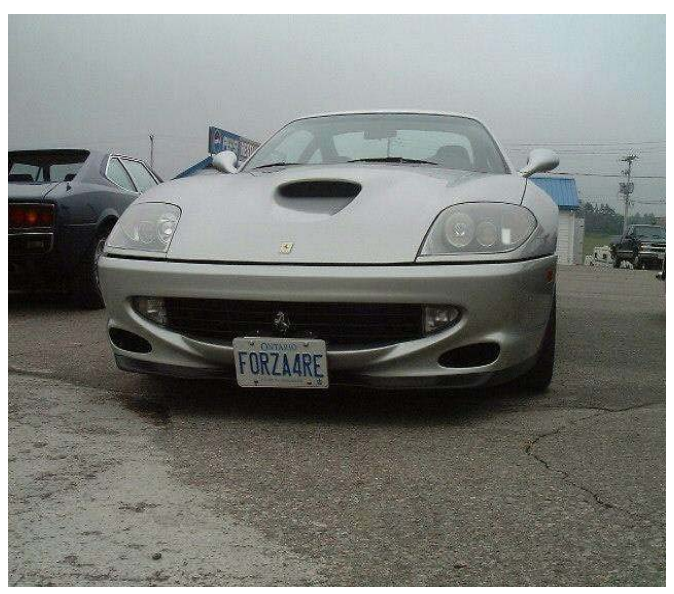

(c)

\section{FORZZLARE}

(d)

Fig. 5 Some License Plates: (a) and (c): Initial images. (b) and (d): Binarization results

Based on these results, the proposed method shows satisfactory and encouraging results make it efficient to cope with some difficult such as variations of light conditions, complex background, and different kinds of license plates.

\section{Concluding Remarks}

We proposed an effective method for LPR system by using the mean shift algorithm and the Fuzzy ARTMAP neural network classifier, which increased the recognition rate of the system. The binarization method provided a good result which confirms to the final recognition rate.

However, the majority of character misclassification problems was due to similar characters, such as 1 and I, 0 
and $\mathrm{O}, 2$ and $\mathrm{Z}, 5$ and $\mathrm{S}$, and 8 and $\mathrm{B}$. Especially, misclassification occurred in the cases of " 8 -- B". The evident solution is the introduction of a priori knowledge for the kind of characters to be recognized (letter or number). However, with this assumption, the LPR process would be country specific.

We are currently undertaking research for extracting key points from the vehicle mask on the basis of license plate position and creating a digital signature for every vehicle model. Moreover, for under-vehicle inspection, it is assumed that a template under-vehicle image for each inspected vehicle has been archived into a database in advance. Based on the incoming vehicle license plate, the respective template image is retrieved from the database and then compared to the one acquired during real-time under-vehicle inspection.

\section{References}

[1] Chang Seok Park, Byeong Man Kim, Byung Hoon Seo, Jun Woo Kim, and Kwang Ho Lee., 4, "Real-time Recognition of Car License Plate on a Moving Car," Korea Society of Industrial Information System, vol. 9, no. 2, pp. 32-43, 2004.

[2] Kwang-Baek Kim and Jae-Hyun Cho, "Recognition System of Car License Plate using Fuzzy Neural Networks," Korea Society of Computer Information System, vol. 12, no. 5, pp. 313-319, 2007.

[3] Kwang-Baek Kim, Young Woon Woo and Choong-Shik Park, "Recognition of a New Car License Plate Using HSI Information, Fuzzy Binarization and ART2 Algorithm," International Journal of Maritime Information and Communication Sciences, vol. 11, no. 5, pp.1004-1012, 2007.

[4] Hans A. Hegt, Ron J. De la Haye, and Nadeem A. Khan, "A High Performance License Plate Recognition System," IEEE International Conference on Systems, Man, and Cybernetics, vol. 5, pp. 4357-4362, 1998.

[5] J. R. Cowell, "Syntactic Pattern Recognizer for Vehicle Identification numbers," Image and Vision Computing, vol. 13, no. 1, pp. 13-19, 1995.

[6] Dorin Comaniciu and Peter Meer, "Mean Shift: A Robust Approach Toward Feature Space Analysis," IEEE Transactions on Pattern Analysis and Machine Intelligence, vol. 24, no. 5, pp. 603-619, 2002.

[7] Gail A. Carpenter, Stephen Grossberg, Natalya Markuzon, John H. Reynolds, and David B. Bosen, "Fuzzy ARTMAP: A Neural Network Architecture for Incremental Supervised Learning of Analog Multi-dimensional Maps," IEEE Transactions on Neural Networks, vol. 3, no. 5, pp. 698-713 1992.

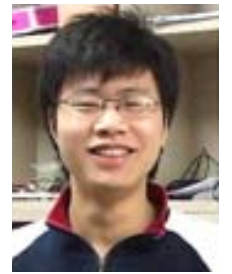

\section{Xiong Xing}

He received his B.S. degree in automation engineering at NanJing Institute of Technology, and M.S. degree from Daegu University. He is currently Doctor Course in the School of Electronic Engineering from Daegu University. His research interests include intelligent control and embedded systems.

Phone : 053-850-4432

E-mail : xiongxing@nate.com

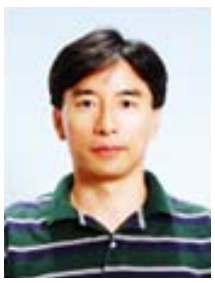

\section{Byung-Jae Choi}

He received his B.S. degree in electronic engineering at Gyeongbuk National University, and M.S. degree and Ph.D. from KAIST. Since 1999 he is working at Daegu University. His research interests include intelligent control and systems.

Phone : 053-850-6633

Fax : 053-850-6619

E-mail : bjchoi@daegu.ac.kr

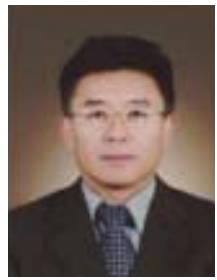

\section{Seog Chae}

$\mathrm{He}$ received the B.S. degree in department of electrical engineering from Seoul National University in 1978, and M. S. and $\mathrm{Ph}$. D. in department of electrical engineering from KAIST in 1980 and 1989, respectively. He joined Kumoh National Institute of Technology in 1983. His research interests include the fuzzy theory and applications.

$\begin{array}{ll}\text { Phone } & : 054-478-7451 \\ \text { Fax } & : 054-478-7449\end{array}$

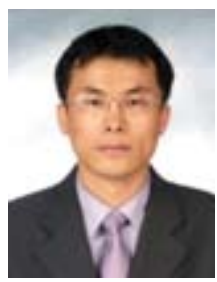

\section{Mun-Hee Lee}

He received his B.S. and M.S. degree in electronic materials engineering at Gyeongsang National University in 1996 and 1998, respectively and Ph.D. degree in multimedia engineering from Pusan National University in 2006. Since 2006 he is working at Daegu Gyeongbuk Development Institute. His research interests include computer vision and IT industry policy.

$\begin{array}{ll}\text { Phone } & : 053-770-5135 \\ \text { Fax } & : 053-770-5059 \\ \text { E-mail } & \text { mhlee@dgi.re.kr }\end{array}$ 